\title{
Leggere il teatro
}

\author{
di Alessandro Quattrone
}

alequattrone58@gmail.com

\begin{abstract}
The theatrical work of Maddalena Mazzocut-Mis, published in the volume Teatro da leggere. Mito e conflitto - with the accompaniment of detailed premises by the author herself - develops in two directions. The first one, looking at the past, leads to the rewriting of works of the literary tradition according to a new point of view. The reworking of famous classical characters relates the distant with the near, allowing us to reflect, among other things, on the function and value of illusions. The second, looking at the present, leads to the presentation of seemingly insignificant characters to highlight the tragic fate of rise and fall or desperate search for a recognized identity.
\end{abstract}

Keywords: Theatre, Myth, Rewriting

La scelta di destinare opere teatrali dichiaratamente alla lettura, come quella fatta da Maddalena Mazzocut-Mis in Teatro da leggere. Mito e conflitto (Le Monnier, Firenze 2020), appare piuttosto insolita. La drammaturgia almeno quella di origine letteraria - trova infatti il suo compimento e la sua ragion d'essere quando altre figure (registi, attori, scenografi, ecc.) estendono il lavoro dell'autore fino alla rappresentazione del testo davanti a un pubblico fisicamente presente. E se il teatro diventa libro? Allora è il lettore ad essere invitato a interagire con l'autore, diventando idealmente regista, attore e scenografo in prima persona: figura attiva, insomma, dal momento che viene offerta una possibilità alla sua immaginazione. "Il lettore impegnato collabora con l'autore», scrive George Steiner. È vero che questo succede sempre di fronte a un'opera letteraria: diamo un volto ai personaggi di romanzo, sentiamo le loro tensioni, entriamo nei loro pensieri. Diventiamo "altri” per qualche momento. Ma lo è a maggior ragione se ad essere letta è un'opera teatrale, dove gli interventi descrittivi dell'autore sono ridotti a qualche breve didascalia, mentre il lettore si ritrova a vedere mentalmente 
azioni, mimiche e gesti dei personaggi secondo la propria libera immaginazione.

Naturalmente i testi teatrali si leggono da secoli, tuttavia sono sempre stati scritti per essere rappresentati. Anche quelli di Maddalena Mazzocut-Mis, sia chiaro, sono nati per la rappresentazione, e sono andati più volte in scena. Converrà quindi riflettere sulla necessità artistica che ha spinto l'autrice a raccoglierli in volume, accompagnandoli con dettagliate introduzioni che descrivono il percorso seguito dall'ideazione alla stesura. Il lettore, che a teatro può conoscere e valutare solo la messa in scena, grazie al libro ha così l'opportunità di visitare il laboratorio della scrittrice e di apprendere qualcosa sul retroterra culturale e sui processi creativi che stanno dietro la produzione di uno spettacolo. E queste informazioni gli permettono non solo di soddisfare tante curiosità, ma anche di comprendere più intimamente la natura delle opere stesse.

Maddalena Mazzocut-Mis arriva alla drammaturgia dopo anni e anni di riflessioni e ricerche sull'estetica (con un'attenzione particolare per il XVIII secolo) confluite in vari volumi di saggistica. Teatro da leggere raccoglie dodici testi tra quelli scritti e rappresentati nel corso di più di un decennio, mentre nel successivo Vuoti a rendere e vuoti a perdere (Le Monnier, Firenze 2021), incentrato su figure e tematiche poetiche, trovano spazio altre quattro drammaturgie, a riprova del fatto che il teatro per l'autrice è sì legato all'occasione, come lei stessa rivela nelle premesse ai testi, ma non certo a una casualità sporadica. Per lei, infatti, il teatro è frutto di una scoperta vocazionale, che l'ha portata alla scrittura appassionata di più dei sedici testi pubblicati in totale.

L’impostazione metodologica propria della saggista, peraltro, che prevede uno studio accurato delle fonti per arrivare all'elaborazione di un proprio 
pensiero, è presente anche nella composizione delle drammaturgie, come si può notare sia nei singoli testi che nelle rispettive premesse. Senza menzionare gli scrittori illuministi, ricordiamo che Montale diceva (citando Tommaso Ceva) che «la poesia è un sogno fatto alla presenza della ragione», e dunque la libertà dell'atto creativo, nell'autrice, è posta sotto la severa sorveglianza della razionalità. Perciò non ci si dovrà mai attendere niente di bizzarro o estroso dalla sua fantasia, ma piuttosto una rigorosa indagine propedeutica alla scrittura, a garanzia dell'attendibilità della vicenda rappresentata.

Senza per questo mortificare l'immaginazione, Mazzocut-Mis procede nel suo lavoro creativo-rielaborativo con un senso di responsabilità di ascendenza manzoniana, convinta di dover rendere conto tanto della plausibilità di ogni espressione quanto della logica dello sviluppo interno dell'azione. Che si tratti di mettere in scena miti classici, personaggi letterari o vite vissute, l'attività preliminare di ricerca e confronto (tipica del saggista) e di riflessione (tipica del filosofo) si uniscono per concepire l'opera. Quando l'opera nasce e si sviluppa, allora l'autrice è pienamente artista. Tuttavia, la saggista-filosofa non si libera della ragione, dopo averla utilizzata, per abbandonarsi arbitrariamente all'immaginazione, ma la tiene sotto la superficie, come il letto in cui scorrono le acque del fiume.

Come si è detto, ogni testo del libro è accompagnato da una ricostruzione della sua genesi, nella quale viene descritto il processo creativo anche attraverso particolari apparentemente insignificanti. Il percorso che conduce dal silenzio alla parola è interessante per questi testi come del resto lo è per qualsiasi opera, se si è desiderosi di capirne i meccanismi. L'autrice, per sua stessa iniziativa, svela aneddoti e circostanze che l'hanno indotta a scrivere su argomenti imprevisti. «Insomma, un breve lavoro di ricostruzione per 
sfatare il mito della creatività come "ispirazione". L'ispirazione c'è... se si studia». Così scrive nella premessa generale.

Per Mazzocut-Mis, quindi, anziché di ispirazione si dovrebbe parlare di occasioni, necessità concrete, scambi di idee con gli amici o anche di specifiche committenze, insomma di tutto quello che può concorrere alla decisione di ospitare nelle proprie stanze interiori non solo i propri fantasmi, ma anche quelli altrui. Cosa che l'autrice fa - per citare solo alcuni - con personaggi come Giocasta, Elena di Troia, Emma Bovary, Candido, Rigoletto, Puck (Mazapegul) e altri a loro connessi, ma anche con persone vere o verosimili, quelle che la cronaca illumina per poco tempo e la grande storia schiaccia e scarta, riducendole a un triste anonimato: ad esempio il dimenticato campione di atletica Emil Zátopek (Volo 903), le vittime e i protagonisti di un attentato terroristico (Cardo rosso), l'impiegata di un ufficio-relazioni col pubblico (e-relazioni, mail pericolose), un'attrice disperata in esilio (Milena).

Il sottotitolo del libro è Mito e conflitto. Tra le opere troviamo infatti sia riproposizioni di vicende mitiche, dove il conflitto è presente in modo archetipico o simbolico, sia vicende storiche, dove i personaggi sono coinvolti in un conflitto reale o verosimile.

I testi presentano i personaggi classici in una luce inedita, mantenendoli comunque coerenti con l'opera originale di riferimento. L'albero è lo stesso, ma i frutti appaiono diversi, essendo il risultato di un'attenta ibridazione. Ł̀ per questo che sentiamo un profumo familiare mentre sperimentiamo un sapore nuovo. Giocasta, Elena, Candido e altri personaggi si configurano nella mente dell'autrice, e nella nostra, con uno scarto dall'originale che ne giustifica la rinascita in una nuova forma.

I classici, come è noto, sono stati spesso fonte di ispirazione per successivi lavori, nel corso dei secoli. Dalla loro matrice sono derivate opere più o meno 
vicine all'originale. Scriveva Edward Young: «È in virtù di un nobile contagio, di una specie di familiarità con i loro scritti, piuttosto che per un furto particolarmente sordido, che possiamo trarre qualche vantaggio da coloro che vennero prima di noi». La presenza del mito (che, come tutto ciò che è classico, ci riguarda perché ci guarda, cioè ci interpreta) non è un limite né una minaccia per gli autori. Certo, potrebbe anche comprimerne l'immaginazione, riducendoli al ruolo di semplici rifacitori, moltiplicatori di varianti senza una vera e propria forza autonoma. Ma un vero autore accresce un patrimonio comune. Ciò che non è "d'autore", invece, ripete il già detto, usa moduli e formule che non attraggono e non sorprendono, lasciando sostanzialmente tutto com'era.

C'è chi mal sopporta l'incombenza del mito sulla propria creatività, e si preoccupa di attaccarlo o di ignorarlo. Non vogliamo qui discutere sulla possibilità effettiva, per un autore, di essere così originale da estraniarsi dall'influsso dei miti, anche indiretto o invisibile, nelle storie che inventa. Per restare alla nostra analisi, diciamo subito che in Maddalena Mazzocut-Mis la tradizione non è un peso da cui liberarsi per scrivere qualcosa di nuovo, anzi può dare inizio a un'opera che sia davvero, e non solo per convenzione o luogo comune, necessaria e attuale. Nel caso dell'utilizzazione del mito e dei classici da parte sua, come vedremo meglio più avanti, la tradizione si rivela stimolante più che ingombrante, nel senso che induce alla creazione di opere che presentano un carattere di novità, pur derivando da altre già esistenti. Nel suo lavoro creativo, infatti, troviamo l'aggiunta di elementi che giustificano l'operazione artistica, permettendo di legare il lontano al vicino, l'estraneo al familiare, l'altrui al proprio.

$* * *$

Il libro è diviso in quattro sezioni: due legate ai classici (Mito e Classico) e due a problematiche contemporanee (Vita oggi ieri domani e Conflitto), ed è 
ricco di proposte. Vale la pena di passare in rassegna le opere raccolte, per avere un'idea della poetica dell'autrice.

In Giocasta la storia di Edipo viene rivissuta appunto dalla regina Giocasta, la moglie-madre, in un monologo straziato e straziante. È questa la novità che fa crescere il personaggio consegnatoci dalla tradizione. La regina si rivolge al figlio-marito, «non per chiedere perdono, non per autoassolversi», ma per rivelare il proprio dramma intimo. Siamo di fronte a una focalizzazione inedita, che si sofferma con acutezza sulla psicologia della donna. Così Maddalena Mazzocut-Mis, sul solco di alcuni predecessori (ad esempio il Cocteau della pièce La macchina infernale) rivitalizza una materia altrimenti destinata a giacere tra le ceneri della nostra memoria culturale. $\mathrm{E}$ la brace che diventa fuoco è tutta nel cuore di Giocasta, che parla non «all'Edipo parricida e vendicativo» ma a quello «incarcerato e ignaro della sorte della moglie e soprattutto dei figli».

Giocasta - quando pensa a Edipo - è innanzitutto una donna che ama, che ha amato e che ha desiderato l'amore, quello che il marito Laio le ha negato, considerandola solo per il suo ruolo di regina da esibire e da violare. "Una notte, Laio, ubriaco, attraversò il palazzo urlando e bestemmiando gli dèi. Si fece aprire le mie porte e si gettò su di me. Nessuna festa ha coperto le mie urla».

La regina è una creatura conflittuale come moglie e come madre, dopo essere stata felice con Edipo sia come moglie che come madre. L'illusione l'ha salvata, la scoperta della verità la rovina. Nel suo monologo è razionale, e indagare è proprio della razionalità. Ma l'indagine non può che condurla alla follia, perché fa emergere la verità, come ogni forma di follia.

Il linguaggio dell'autrice ha la solennità poetica della tragedia antica. «Non parlo per farti del male e non sarà lo strazio a trovare le parole, ma la pietà 
di una madre che dice mentre nasconde». Mazzocut-Mis è brava a simulare il tono dei suoi autori-fonte (da Sofocle all'Euripide delle Fenicie, per arrivare a Seneca). Ad esempio, ne riproduce con eleganza la pensosità gnomica («Ci si illude della propria eternità, quando nulla turba il giorno»; «Si muore sempre troppo presto o troppo tardi»). E poi cita e parafrasa, accosta parole inventate a parole ritrovate, mantenendo unità di stile e di tono nella sua mescolanza verbale. Sembra quasi una discendente degli antichi tragediografi che abbia conservato nel patrimonio genetico tracce evidenti dei progenitori.

In letteratura i processi di ibridazione sono delicati e rischiosi: potrebbero non riuscire, producendo frutti dallo sgradevole sapore di artificioso. $\mathrm{Nel}$ nostro caso l'autrice opera con ponderazione, evitando sia la combinazione posticcia sia la caduta nella semplice imitazione.

Per usare le categorie di Genette, quelli di Mazzocut-Mis sono ipertesti che richiedono la conoscenza degli ipotesti. D'altra parte, tutta la storia della letteratura è infarcita di riferimenti intertestuali più o meno espliciti e più $o$ meno volontari. Alla fine conta, come si è detto, la capacità di aggiungere qualcosa all'esistente, fosse solo uno sguardo. E in Giocasta tutti - anche il marito Laio, i figli Eteocle e Polinice, le figlie Ismene e Antigone - sono visti con gli occhi della protagonista femminile, e questo è già notevole.

«Uomo e bambino mio. Abbandonato. Mio bambino e uomo. Dimenticato. Io ti proteggerò e ricorderemo insieme». Così la donna si rivolge a Edipo colpito dalla sventura, mescolando parole di madre e parole di sposa, senza poterle - e forse senza nemmeno volerle - tenere distinte.

Maddalena Mazzocut-Mis tratta il tema della bellezza femminile, intesa come fonte non di serenità ma di angoscia, in un testo contenuto nel libro (L'eco di un fantasma, ispirato soprattutto dalla lettura di Euripide e della sua Elena) oltre che in un'altra opera esclusa dalla pubblicazione (Jezabel, 
rivisitazione del romanzo di Irène Némirovsky). L'argomento, per un'autrice che è anche docente di estetica, è ovviamente di grande interesse. Colpisce però apprendere come la scintilla che ha incendiato la sua immaginazione sia stata una trasmissione televisiva di qualche anno fa ("Velone"), in cui a esibirsi in una gara di bellezza erano signore anziane e spesso in forte sovrappeso, ben distanti dal modello originario delle note "Veline" televisive. Guardare la trasmissione e pensare a un'Elena vecchia, non più bella, spudorata, per lei è stato facile.

Ma le questioni che la figura di Elena - giovane o vecchia - solleva non sono legate solamente al tema della bellezza e del suo potere ambiguo, fascinoso e terribile: sono anche di natura etica, visto che la fuga da Sparta potrebbe essere interpretata tanto come conseguenza di una complicità delittuosa con Paride quanto come espressione di rassegnazione inerme alle sue mire. Nella premessa al testo, infatti, la drammaturga ci informa: «Alcuni enigmi del personaggio di Elena mi affascinavano da sempre: era stata rapita da Paride o era scappata di sua volontà? Era vittima del volere degli dèi?».

È giustificato il controcanto della severa e accigliata Ecuba, madre di Paride, chiamata a commentare le azioni e le parole dell'irresponsabile Elena? Non sarà che, alla fine, è inevitabile che la vita si presenti con entrambi gli aspetti, e che ci sia sempre qualcuno a ridimensionare chi è sovradimensionato? Forse gli esseri umani attribuiscono troppo valore alla bellezza, che di per sé è transitoria, concedendole il privilegio di poter infrangere l'ordine sociale e familiare, e persino quello politico, sempre con una giustificazione a portata di mano.

Come Giocasta, L'eco di un fantasma è una tragedia lirica: anch'essa costituisce il libretto di un'opera musicale di Azio Corghi, ma ha una sua leggibilità autonoma. Il discorso fatto per Giocasta può essere riferito anche a quest'opera per quanto concerne la procedura compositiva, ma non per gli esiti. Qui siamo di fronte a un ipertesto che rivisita il personaggio originale liberandolo dalla tradizionale aura mitica e incantevole e degradandolo fino 
all'umiliazione, senza sconfinare nei territori del comico e del parodistico. Elena è una donna che con gli anni invecchia e imbruttisce, che non accetta di perdere il potere della seduzione, e con le vesti di una signora di provincia degli anni '60-'70 cerca nell'adulterio (e il pensiero non può che andare anche a Emma Bovary, protagonista di un'altra opera di Mazzocut-Mis) i piaceri della vita estetica kierkegaardiana, banalizzandoli. Questa Elena è tutt'altro che una creatura superiore, anzi si offre con facilità: è sfrontata e impudica. Con un giovane amante arriva a compiere atti osceni in pubblico. Non è per niente splendida, anzi appare volgare e squallida nelle sue iniziative. Nella passione carnale non trova slancio ed esaltazione: la sua avventura è priva di emozione e non porta a scoperte meravigliose. L'unico suo godimento è nell'esperienza dell'irresponsabilità. «Bellezza nasce per peccare. Altrimenti non è Bellezza». Seduttrice e amante, in realtà Elena non ama l'altro, e non ama nemmeno l'amore: ama il potere, e la bellezza è un potere insidioso, che però è destinato a scemare con il tempo.

La donna dà la colpa agli altri delle proprie azioni più vergognose. «La bellezza non è una colpa. Perché hai lasciato che mi rapisse?» dice a Menelao, puntandogli il dito. Secondo lei il marito la lascia rapire, Paride la rapisce. Dunque, lei non c'entra niente con la morale e l'immoralità.

L'Elena di Mazzocut-Mis ha un suo "Menelao" da tradire e un suo "Paride" con cui fuggire in un tentativo estremo, e per certi versi misero, di entusiasmo. Paride per lei è solo uno strumento per potersi sentire splendida. Elena in realtà è rapita non da un principe straniero, ma dal proprio intimo sogno di eccezionalità. Il suo Paride non è che un eroe di cartapesta, un eroe banale. E non c'è niente come l'ombra della banalità che possa oscurare la Bellezza. La grande passione non si può accendere in ambienti squallidi, non si può nutrire di momenti meschini. Tutto deve circondarsi di un'aura luminosa e scintillante come l'illusione. La banalità è nemica dell'illusione, e quindi della passione. Per Elena tutto è accettabile tranne l'ordinarietà. E invece il suo Paride è ordinario, e quindi deludente: «Da te mi aspettavo qualche vizio o 
qualche virtù. Se tu fossi stato un eroe, se tu mi avessi portato via dalla provincia, forse avrei potuto amarti».

C'è poi da considerare il rapporto di Elena con la figlia. Se con gli amanti è un po' Emma Bovary, con lei (Ermione) è un po' come la Jezabel di Némirovsky: crudele e sprezzante. La sfida e la odia perché in lei vede il suo contrario; e arriva a rubarle il ragazzo per sentirsi superiore. Dal canto suo la figlia, patologicamente, cerca di essere l'anti-madre. Non compete. Non vuole superarla, ma ucciderla dentro se stessa, per liberarsi della sua eredità.

L'eco di un fantasma, insomma, ci dice che viviamo di fantasmi, tra i fantasmi, essendo anche noi fantasmi per i fantasmi che ci circondano: fattezze e identità sono mutevoli, viviamo - come dice Pirandello - della vita che ci danno gli altri, mentre tutto cambia in noi e attorno a noi, riducendoci a immagini della nostra mente e di quella altrui. Siamo e non siamo, siamo e svaniamo, svaniamo e ritorniamo, per svanire definitivamente. «La bellezza è fantasma».

Nel suo viaggio alla riscoperta dei classici, Maddalena Mazzocut-Mis sembra prendere particolare gusto a mettere in scena Voltaire come personaggio accanto alla sua celebre creatura, Candido. Il filosofo, con scintillante e studiata ironia, manovra, critica e corregge quest'ultimo fino a confonderlo e a scoraggiarlo, ma sempre provvisoriamente, perché Candido trova comunque un motivo illusorio per riprendere la sua ricerca della felicità. «Candido è la vittima di un carnefice inesorabile», commenta l'autrice. Voltaire, dunque, è un torturatore del suo personaggio: interviene in continuazione per dimostrargli l'insensatezza dei suoi desideri. Ma Candido, imperterrito e ostinato, si nutre di illusioni, che per lui sono naturali matrici di senso, così riesce a mantenersi saldo nonostante tutte le sventure e le 
disavventure a cui va incontro, dimostrando che l'ingenuità in fondo può essere un formidabile strumento di resistenza ai colpi della sorte.

Candido è sospinto di qua e di là dagli eventi, si accende e si spegne, gioisce di una gioia sempre fatua e soffre di una sofferenza sempre fugace, facile alla speranza e alla disperazione, padrone delle proprie illusioni, non del proprio destino. La drammaturgia effervescente di Mazzocut-Mis riproduce il ritmo del racconto originale attraverso il movimento del povero Candido, che si sposta da un luogo all'altro alternando uno stato d'animo all'altro. Candido cerca nel viaggio «una liberazione da un destino opprimente e infausto». Il migliore dei mondi possibili non si trova da nessuna parte, ma lui deve e vuole credere che esista, come l'isola di cui ha bisogno il naufrago. Quell'isola, alla fine, sarà il proprio giardino, dove potrà considerarsi salvo, accontentandosi dell'amata Cunegonda (a lungo persa di vista e ritrovata ormai «brutta, senza denti, arcigna e inacidita») e di un'assenza di eventi tutto sommato consolante.

Se durante il viaggio ha conosciuto l'essenza precaria e disperata della vita, che ha messo a dura prova il suo candore innato, nel coltivare il giardino Candido può continuare a coltivare anche l'ingenuità, per di più impunemente. Tuttavia, secondo Mazzocut-Mis, «rinchiudersi entro steccati rassicuranti e solipsistici significa anche rinunciare alla passione e approdare sulle rive desolate della noia». Solo la curiosità ci rende vivi, anche se porta con sé un aumento di vulnerabilità. Il giardino è un rifugio, sì, ma un «rifugio infelice». E così Voltaire non può che esortare ironicamente il suo incorreggibile personaggio a starsene al sicuro. "Coltiva, mio caro amico, coltiva. Il più insignificante degli orti possibili!».

Nella rivisitazione di Madame Bovary Mazzocut-Mis si ispira solo indirettamente al romanzo (che, come è noto, alla sua uscita suscitò uno 
scandalo), preferendo concentrare l'attenzione su Flaubert e sul processo da lui subito per oltraggio alla morale pubblica e religiosa, oltre che ai buoni costumi. L'autrice presenta sulla scena lo scrittore francese, il suo avvocato e il pubblico ministero (con il compito di incarnare le idee del pubblico), oltre alla stessa Madame Bovary e ad altri personaggi del romanzo.

È proprio la presenza di Flaubert a costituire il motivo di interesse principale dell'opera e a permettere a quest'ultima di dilatarsi, spingendosi ben oltre i limiti di un adattamento del romanzo per il teatro. Come spiega l'autrice nella premessa, «l'aggiunta di Flaubert sulla scena ha la funzione di proporre una rilettura del romanzo attraverso gli occhi dell'autore. Occhi sinceri, perché le parole di Flaubert sono sempre tratte da lettere e scritti di suo pugno».

Di passaggio gioverà osservare come Mazzocut-Mis - rispettando in questo modo la forma mentis di Flaubert, notoriamente ossessionato dalla perfezione dello stile e dalla ricerca del mot juste - scelga di mescolare le battute originali della drammaturgia con quelle scritte sia dal Flaubert-uomo che dal Flaubertautore, evitando di parafrasarlo o di attribuirgli espressioni inventate. Ogni parola è pesata e ha un suo valore specifico, come avviene sia nell'ambito letterario che in quello giudiziario.

La madame Bovary di Mazzocut-Mis è inscindibile dal suo creatore, che non per nulla si identificava in lei (Madame Bovary c'est moi). «Il processo diventa un modo di sottoporre a giudizio, insieme a Emma Bovary, lo stesso Flaubert», chiarisce in premessa l'autrice. E dunque la sua opera indaga il rapporto tra romanziere e personaggio, nonché tra l'anima maschile e l'anima femminile. Come fa il suo Voltaire con Candido, anche il suo Flaubert condanna e tortura il proprio personaggio. Lui uscirà assolto dal processo, Emma invece si rivelerà in tutta la sua miseria di anima votata al kitsch, nonostante viva la tragedia del disfacimento psicologico e morale. Sia Candido che Emma Bovary hanno un insopprimibile bisogno di illudersi. Ma mentre Candido, pur passando da un'illusione all'altra, riesce a sopravvivere 
trovandovi energia e conforto, Emma è travolta dai propri sogni inconsistenti. Flaubert la detesta. Disprezza le ristrettezze e le vanità della mentalità borghese, di cui Emma è un perfetto esempio provinciale. Ma anche lui, il suo creatore, è borghese e provinciale, in fondo. Dunque, è se stesso che Flaubert detesta, ed è di se stesso che vorrebbe liberarsi, in realtà, scrivendo di lei.

Maddalena Mazzocut-Mis, come si è detto, mette al centro della sua Madame Bovary il rapporto tra autore e personaggio. In uno dei primi scambi di battute tra Flaubert e il suo avvocato evidenzia la stretta somiglianza tra i due. Flaubert dice che «Emma ha tutto», ma l'avvocato precisa che «ha tutto ma nulla di ciò che vuole. Ecco dove ti somiglia, ti lamenti in continuazione». Lo stesso Flaubert conferma più avanti: «Tutto quello che amo in quel libro non c'è». Perché lui è un descrittore: non manifesta semplicemente il suo disprezzo per il provincialismo, ma lo illustra e lo scandaglia, per arrivare alla verità. E in Flaubert l'amore per la verità è tale da fargli trovare il materiale per una scrittura perfetta nella mediocrità che si consuma fino alla degradazione. Analogamente a Baudelaire, lo scrittore normanno raccoglie i suoi fiori stilistici nel campo del male, cioè della bruttezza morale e psicologica. «Il mio romanzo è magnifico, direi perfetto. La mediocrità è nauseabonda. Lo sono i suoi personaggi. Emma è nauseabonda».

Nel corso del processo l'avvocato difensore punta tutto sulla presentazione di un Flaubert-autore da intendersi come rivelatore della realtà: un difensore della donna, in fondo, un moralista sensibile, che mostra la realtà a chi la ignora. Ma il Flaubert-personaggio nega che le cose stiano così: lui, attraverso madame Bovary, si è solo liberato della scialba volgarità e della presuntuosa insulsaggine che sente dentro di sé. «Non mi sono mai vergognato di lei. Mi vergogno di me stesso. Solo di me stesso! Della mia doppiezza, della noia che ho di me. La mia persona mi ripugna tanto che me ne sento nauseato». Mentre il suo avvocato cerca di far passare il romanzo per un'opera dal carattere morale, Flaubert si preoccupa di difendere la perfezione estetica del suo 
personaggio, costruito sulle imperfezioni umane. In altre parole, difende l'arte, non la vita.

Tutti attaccano la donna, che è ormai una creatura abbandonata e indifesa ma non ha niente di romantico, di teatrale, di eroico. Avere tutti contro non la innalza dal terreno della mediocrità su cui ha sempre camminato. Lei che ha sognato gli splendori della libertà, gli slanci romantici, l'avventuroso superamento dei limiti, si ritrova confinata nella propria solitudine assoluta. La sua vicenda si svolge freddamente, non avendo i caratteri tipici del melodramma. Ed è proprio per questo che l'autrice finisce con il simpatizzare per lei. Non potendo redimere e risarcire Emma come donna, Mazzocut-Mis si dedica al riscatto del personaggio. Lo libera dal carico negativo addossatole dal suo autore, che forse si era voluto liberare a sua volta proiettando in lei la propria ombra. La vittima, predestinata dal suo creatore alla sventura e al disastro, alla fine suscita sentimenti di empatia e generosità sia nell'autrice che nello spettatore.

Così nel corso dell'opera Flaubert da accusatore diventa accusato, da persecutore perseguitato. E da creatore onnipotente, creatura colpevole. Ma non fa che ribadire una verità paradossale: il personaggio è vero, l'autore è falso. «Emma sono io».

$$
* * *
$$

Delle due sezioni Mito e Classico, oltre alle opere su cui ci siamo soffermati, fanno parte anche Un sogno (riscrittura di Sogno di una notte di mezza estate) e Il re si diverte, a testimonianza del fatto che l'autrice ama frequentare i classici antichi e moderni. Ma nelle altre due sezioni del volume (Vita oggi ieri e domani e Conflitto) non mancano opere incentrate su tematiche di più 0 meno recente attualità. 
E-relazioni, mail pericolose offre un saggio di plurilinguismo contemporaneo, che dimostra tutta la versatilità della scrittrice, capace di inventarsi una forma di teatro epistolare evocando nel titolo il romanzo di Laclos, il settecentesco Le relazioni pericolose. Qui però non è in gioco la vita estetica in senso filosofico, ma solo la sua declinazione cosmetica. Le due opere hanno in comune il cinismo. In e-relazioni, mail pericolose non c'è il culto della vittoria narcisistica, ma la necessità commerciale di superare la resistenza e la diffidenza del cliente. E per farlo si ricorre alla beffa di lontana matrice boccaccesca, che prevede il trionfo dell'intelligenza, nella sua forma astuta, sulla stupidità.

In questa singolare opera la satira di un certo mondo commerciale colpisce tanto i venditori quanto gli acquirenti, gli uni spregiudicati e gli altri ingenui. Il linguaggio è degradato a gergo pseudospecialistico, ricco di anglismi che dovrebbero far pensare alla estrema modernità dell'azienda e impressionare il cliente. Il vero protagonista della pièce è proprio il linguaggio con la sua potenza retorica, che ricorre ai tecnicismi allo scopo di escludere l'interlocutore, di diminuirlo, di ridurlo a povero postulante, ad aspirante, a sognatore da punire per la sua credulità.

L'umorismo si esprime nelle sue diverse sfumature, da quelle più velatamente ironiche a quelle più pesantemente sarcastiche. Qui non c'è traccia di miti e tragedie antiche, né di personaggi così celebri da essere diventati quasi familiari. C'è piuttosto una figura comune al giorno d'oggi: una laureata (in archeologia) costretta ad adattarsi a un lavoro che non fa per lei.

La giovane donna non riesce a calarsi perfettamente nel ruolo, che in sostanza è quello di alimentatrice di illusioni. D'altronde, dalla rassegna di personaggi che contattano l'ufficio, pare quasi che gli illusi si meritino l'inganno, anzi, che ne abbiano bisogno. Uomini e donne in crisi, affetti da 
ossessioni e disturbi legati all'aspetto fisico, le si rivolgono di volta in volta con fiducia, rabbia o disperazione. La quarantenne protagonista, via e-mail, si relaziona con loro sotto tante vesti e tanti nomi, usando caratteri, registri linguistici e toni diversi a seconda del caso. Ha un indirizzo di posta elettronica per ogni identità che assume: addetta alle relazioni con i clienti, dottoressa specialista, amica, figlia e aspirante ricercatrice universitaria. Passa così in continuazione dalla necessità di mostrarsi fredda e professionale a quella di abbandonarsi a un calore emotivo disarmato e inquieto. Come dottoressa il personaggio dà magniloquenti consigli di cura del corpo e dello spirito ai clienti più perplessi, scivolando facilmente nel biasimo moralistico a scopo intimidatorio; come addetta alle relazioni affronta i problemi con risposte formali o interlocutorie; come figlia e amica alterna verità e finzione, drammatizzando e rassicurando; come ricercatrice esprime tutta la propria fiera disperazione.

La pièce è spassosa e seria, ironica e caustica, e ci permette di conoscere l'animo di chi, lavorando nella precarietà più assoluta, rimanda il proprio sogno (la carriera culturale) a data da destinarsi, e intanto alimenta quello altrui (la forma fisica) con una ipocrisia tanto sarcastica quanto necessaria. Il personaggio, nella sua evoluzione, arriva a esprimere - schizofrenicamente la verità cruda e irrimediabile nelle e-mail private, e quella altrettanto cruda ma speranzosa nelle e-mail di lavoro.

Conclusione: si può solo fingere, a questo mondo. E il compito del teatro è smascherare mostrando le maschere. Come sempre è accaduto e sempre accadrà. Non a caso e-relazioni, mail pericolose apre la sezione del libro intitolata Vita oggi ieri domani. 
Della sezione Vita oggi ieri domani fa parte anche il Monologo a due, dove i personaggi (un gatto umanizzato e il suo padrone) mangiano e discutono di regimi alimentari.

È giusto mangiare carne? Il dibattito oggi è molto diffuso, ed è fondato sostanzialmente su una domanda: anche gli animali rientrano sotto la protezione dell'etica, in ogni caso, senza eccezioni? Maddalena Mazzocut-Mis in premessa scrive che «la pièce non conclude, non sceglie. Piuttosto evidenzia anche le contraddizioni. [...] Il teatro, la letteratura possono e devono aiutare il dibattito». Prima che l'opinione pubblica e poi la giurisprudenza assumano decisioni nette, il dialogo (meglio ancora se teatrale) fornisce spunti, riflessioni, tesi e antitesi su cui soffermarsi.

Che lo spettatore, andando a teatro, possa e debba trovare motivi per pensare e orientarsi è idea antica (citiamo, uno per tutti, il riformista Terenzio nella Roma conservatrice del suo tempo). Ma le scelte personali non fanno ancora giurisprudenza.

Il dibattito è ancora ampio a livello filosofico, scientifico, politico e giuridico, e c'è bisogno di stimoli alla riflessione che aumentino la lucidità delle opinioni.

Ricorrendo a un facile gioco di parole, possiamo dire che il "senso" della vita si costruisca sui "sensi"? I sensi sono così importanti da permetterci di entrare non solo in contatto ma anche in relazione col mondo? E quanto contano per raggiungere un'attendibile coscienza di sé stessi?

Maddalena Mazzocut-Mis si stava occupando di un progetto che prevedeva un'indagine sul ruolo dei sensi nel teatro (e peraltro in precedenza aveva studiato il rapporto tra la vista e il tatto), quando ha cominciato a scrivere la pièce Per non vedere, anch'essa inclusa nella sezione Vita oggi ieri domani. In 
questo caso la situazione che rappreseTnta è poco comune, benché possa essere universalizzata e attualizzata.

Prendendo spunto dall'opera di Maeterlinck I ciechi, l'autrice (che ha scritto l'opera a quattro mani con Tancredi Gusman) ambienta la vicenda «su un'isola, su una terra di nessuno, che protegge i personaggi da quel che accade sul continente e li rende altresì prigionieri», come precisa lei stessa nell'introduzione.

Un gruppo di non vedenti si ritrova per fare un'escursione in un bosco. Tra loro le posizioni intellettuali e gli atteggiamenti spirituali sono diversi, e dipendono dal modo di concepire la cecità. C'è chi non vede dalla nascita, ma anche chi ha deciso che è meglio non vedere. C'è poi chi afferma di vedere, e anche chi finge di vedere per poter esercitare una funzione di guida: il prete che accompagna il gruppo.

Si parla di sensi, ma come non sovrapporre al verbo vedere il verbo capire, pensando alla vita? La scomparsa del prete creduto vedente (un benefattore che è convinto di poter aiutare gli altri regalando illusioni) costringe gli altri a regredire verso il fondo animalesco della propria vita, alla ricerca di una possibilità di sopravvivenza, e a fare i conti con una paura a un tempo fisica e metafisica, che diventa matrice di ragionamenti e discussioni di stampo esistenziale. La deprivazione sensoriale ha effetti sull'atteggiamento psicologico nei confronti della vita, ma anche sulla concezione filosofica che se ne sviluppa.

Uno dei temi principali dell'opera è il valore delle illusioni. Credere che qualcuno possa guidare gli altri è solo un'illusione che fa coincidere la fiducia con la salvezza. Uno dei ciechi, appassionato di fotografia, è forse la figura più emblematica della pièce: crede alle illusioni e non alle cose vere. Fotografa seguendo le descrizioni del prete, che utilizza la memoria e la fantasia come sostituti della vista.

Per non vedere è un'indagine sulla percezione sensoriale e una grande allegoria della vita, capace di creare tensione emotiva e allo stesso tempo 
filosofica. In definitiva, è il pensiero umano ad essere cieco. «Non puoi fare il fotografo con gli occhi degli altri!»: è il rimprovero di un personaggio a un altro, ed è come se il realismo dei sensi rimproverasse l'idealismo del desiderio.

Nella sezione Conflitto, che si apre con Cardo rosso, elaborazione teatrale di un grave fatto di cronaca (l'irruzione terroristica del 2002 nel teatro Dubrovka di Mosca, con la presa in ostaggio di attori, musicisti e spettatori e tutte le tragiche conseguenze che ne derivarono), Mazzocut-Mis mette in scena tante storie individuali e circoscritte per parlare di una barbarie sempre possibile.

Il teatro (inteso come spazio) è il luogo della rappresentazione della verità, che è intrinsecamente violenta, perché spazza via tutte le false e comode certezze su cui ci adagiamo. E Cardo rosso è teatro nel teatro, nel senso non del metateatro, ma del luogo reale della tragedia, che diventa luogo simbolico della possibilità di un contatto profondo tra estranei, vittime e spettatori.

La rappresentazione, attraversata da interrogativi inquietanti, è un modo per rendere le vittime non giustiziere ma giudici, e per far conoscere certi sentimenti brucianti (come l'odio incontenibile, il terrore accusatorio e la paura irredenta) a chi non ne è mai stato sfiorato per una sorta di immunità dovuta a mera fortuna o a banale - e magari colpevole - distrazione.

Dai dialoghi dei tanti personaggi si capisce che le ragioni degli uni e degli altri a volte sono solo reazioni agli uni e agli altri, e i ragionamenti, più che sulla inoppugnabilità della logica, si fondano sui diritti morali - veri o presunti - derivanti dal dolore.

Se alla tragica notizia di cronaca possiamo opporre i nostri sbrigativi commenti (e il nostro naturale sollievo di lucreziana memoria), di fronte all'arte invece, secondo Mazzocut-Mis, siamo indotti a una partecipazione 
sentimentale e intellettuale che non potrà rimanere senza esito: «I fatti sono solo un punto di partenza; non volevo realizzare un'opera di ricostruzione documentaristica. Si tratta, piuttosto, di finzione e di teatro, dove l'elemento drammatico, che non sfocia nel patetico, rimanda agli accadimenti solo in modo traslato, per diventare tragedia corale, tragedia di emarginazione e di cecità».

Gente comune, soldati, terroristi: tutti, dai più frivoli ai più severi, dai più idealisti ai più egocentrici, hanno le loro ragioni e le loro spiegazioni, tutti ritengono di sapere cosa sia giusto, e intanto sono tutti parte della tragedia non solo con il loro presente ma anche con la loro storia personale. La vicenda, infatti, si sviluppa su piani temporali diversi per ricordarci che il passato è proprio ciò che non passa mai, e il presente non è altro che passato non trascorso. Ogni personaggio racconta e si racconta secondo la propria ottica. «Lo sguardo dello spettatore è quindi la risposta di fronte alla nudità dei racconti, che svelano un mondo dove la vittima e il carnefice si inseguono e si sovrappongono».

Cardo rosso è un grande contenitore di crudeltà, tenerezza, ingenuità, incomprensione, rozzezza, sogni e disprezzo, in un contesto in cui spiccano le voci delle madri, "carnefici e vittime" anch'esse, e quella di un giovane soldato in crisi: umano, quindi inadeguato al ruolo, dato che dove c'è conflitto prevale il ruolo (la divisa) sulla pura umanità.

Leggendo Milena, sempre all'interno della sezione Conflitto, ci troviamo di fronte a una protagonista di cui non sappiamo nulla, se non che è una giovane attrice e vive in esilio in un piccolo appartamento assieme a una drag queen. Un tempo, nel suo paese d'origine (governato da un regime autoritario), è stata ricca e famosa, perché ha condiviso la sua fortuna con un noto gerarca. $\mathrm{E}$ ora, come scrive l'autrice nella premessa, «vive rimpiangendo una felicità e 
una vita incomprensibile ai suoi interlocutori», raccogliendo "cocci di ricordi che ricompone: i suoi grandi amori, il successo, la guerra, la morte».

La storia è ispirata alla vita vissuta; quindi, come in Cardo rosso, si presenta la necessità di trasformare in arte la cronaca. Alla maniera di Manzoni, Mazzocut-Mis immagina - sulla base delle documentazioni consultate - cosa potrebbe aver detto, pensato e sentito una donna passata dall'altezza alla bassezza, dal successo all'anonimato. In lei si sviluppa il dramma del capro espiatorio che, a causa della guerra civile, ha dovuto subire rappresaglie e abbandoni, e compiere la sua discesa agli inferi dopo il trionfo.

«Milena incarna la profonda e inalienabile rabbia di chi sa che la guerra ha rubato il suo destino», spiega l'autrice. La disgrazia la rende esemplarmente umana: mutatis mutandis (cioè sostituendo all'azione divina quella della fortuna), come nel Napoleone manzoniano in lei è più evidente l'orma del destino, cioè di una forza - storica, imprevedibile, violenta - che non tiene in alcun conto la volontà umana, riducendo l'individuo a una misera combinazione di desideri e di rimpianti.

La giovane donna in esilio è un'umile cameriera, nel suo paese era una grande attrice. Ma un giorno il contatto con il potere l'ha tentata e corrotta, e allora, credendo di poter essere un soggetto, è diventata un oggetto - uno strumento - nelle mani altrui. Ora vorrebbe solo poter essere ciò che è stata, riavere il volto che la guerra ha reso irriconoscibile. Anche lei, come tanti personaggi del teatro di Mazzocut-Mis, ricerca disperatamente una identità definita, senza cui non è possibile vivere sensatamente. $E$ in mancanza di una identità precisa ne assume tante altre approssimative, parziali - tutte insufficienti - illudendosi di poter raggiungere l'unità con la loro somma.

Accanto alla drag queen, che di trasformazioni vive, Milena di trasformazioni muore. 
Volo 903 presenta un'altra storia ispirata a una vicenda di vita vissuta, quella di Emil Zátopek, un grandissimo atleta passato, per decisione politica, dall'esaltazione alla degradazione, dalle medaglie d'oro vinte alle olimpiadi al mestiere di netturbino, per di più sorvegliato da un delatore. "Così nasce Volo 903: dall'eroica esaltazione di un mito, alla quotidiana sopravvivenza. Dallo Zátopek atleta imbattibile, allo Zátopek umiliato dallo Stato socialista che lo aveva osannato».

Sembra che all'autrice stia molto a cuore l'imprevedibilità dei casi umani, con la loro dinamica di ascesa e caduta. Il destino ci stupisce prima perché a vincere è chi sembrerebbe non avere le caratteristiche per riuscirci («Corre male e vince [...] è brutto quando corre [...] è pesante, scomposto»), poi perché a essere umiliato è un uomo che non lo meriterebbe, non solo per i suoi successi, ma anche perché generoso e idealista. "Ho imparato allora che la vittoria va sempre condivisa", dice l'atleta rievocando la propria storia di corridore sulle lunghe distanze. La sua vita, ai tempi dell'attività sportiva, è stata corsa, resistenza, numeri. La sua sofferenza è diventata sacrificio al dio del movimento, per avere in cambio la vittoria, che per lui voleva dire soprattutto superamento dei propri limiti.

Il corridore durante la gara è solo contro tutto e tutti. Sperimenta la condizione umana nella sua purezza astorica. Ma quando torna alla vita sociale, sono altre le esigenze: il mondo chiede qualcosa che non si vorrebbe o non si potrebbe dare. Adesso il limite non è il proprio essere, non è la propria volontà: è l'essere altrui, la volontà altrui; in altre parole, è la concretezza degli altri uomini al servizio di un regime politico totalitario. Non si può vivere nel vento, essere nel vento. Si è nella storia, dove la purezza si disperde e gli slanci sono guardati con sospetto. Perciò il campione Zátopek, divenuto uomo comune, da vincitore viene ridotto a vittima. Il suo corpo non può più volare. 
Sulla scena, accanto all'atleta troviamo un ottuso servitore dell'ideologia, che prende alla lettera ciò che è metaforico e per metaforico ciò che è letterale, perché tutto deve rientrare in una visione preordinata, e lo strumento più comodo per farlo è interpretare, non importa se in modo autentico o meno. Zátopek gli parla con il cuore puro, ma il delatore interpreta con il cuore contaminato. Il conflitto è unilaterale. Il delatore perde - moralmente perché combatte; l'ex atleta - moralmente - vince perché non combatte. Vince perché riesce a sorridere, nonostante tutto.

Zátopek, scrive l'autrice, «convinto di avere incontrato la fortuna e di avere vissuto una vita piena di soddisfazioni, sorride. Sorride anche quando, come netturbino, passa i sacchi al suo compagno, correndo, ancora una volta, dietro il camion della spazzatura».

\section{$* * *$}

Nella volontà dell'autrice, che nella premessa al volume ci invita a fare amichevolmente un "pezzetto di strada insieme», c'è l'idea di farci accompagnare lungo il percorso da personaggi che forse non conoscevamo o che credevamo di conoscere. E alla fine della lettura del volume si ha davvero la sensazione di aver fatto un viaggio che ci ha permesso per qualche ora di vivere altre vite, sentire altri sentimenti, pensare altri pensieri. Rimanendo chiusi nella nostra stanza, con un libro in mano.

\section{Nota bibliografica}

CHODERLOS DE LACLOS, Pierre, Le relazioni pericolose, tr. it di M. T. Nessi, Garzanti, Milano 2007.

COCTEAU, Jean, La macchina infernale, tr. it. di M. Zini, Einaudi, Torino 1989.

GENETTE, Gérard, Palinsesti. La letteratura al secondo grado, tr. it. di R. Novità, Einaudi, Torino 1997. 
MANZONI, Alessandro, Poesie e tragedie, Garzanti, Milano 1990.

MAZZOCUT-MIS, Maddalena, Teatro da leggere. Mito e conflitto, Le Monnier, Firenze 2020.

—, Vuoti a rendere e vuoti a perdere, Le Monnier, Firenze 2021.

NÉMIROVSKY, Irène, Jezabel, tr. it. di L. Frausin Guarino, Adelphi, Milano 2007.

OTT, Christine, Montale e la parola riflessa, FrancoAngeli, Milano 2006.

PIRANDELLO, Luigi, La vita che ti diedi, Mondadori, Milano 1951.

STEINER, George, I libri hanno bisogno di noi, tr. it. di E. Lana, Garzanti, Milano 2020.

YOUNG, Edward, Congetture sulla composizione originale, tr. it. di F. Carboni, Book editore, Ro Ferrarese 2008. 